\title{
FINITE RANK TOEPLITZ OPERATORS ON THE BERGMAN SPACE
}

\author{
DANIEL H. LUECKING
}

(Communicated by Joseph A. Ball)

\begin{abstract}
Given a complex Borel measure $\mu$ with compact support in the complex plane $\mathbb{C}$ the sesquilinear form defined on analytic polynomials $f$ and $g$ by $B_{\mu}(f, g)=\int f \bar{g} d \mu$, determines an operator $T_{\mu}$ from the space of such polynomials $\mathcal{P}$ to the space of linear functionals on $\overline{\mathcal{P}}$. This operator is called the Toeplitz operator with symbol $\mu$. We show that $T_{\mu}$ has finite rank if and only if $\mu$ is a finite linear combination of point masses. Application to Toeplitz operators on the Bergman space is immediate.
\end{abstract}

\section{INTRODUCTION}

In classical function theory of the unit disk, Toeplitz operators were defined on the Hardy space $H^{2}$ by $T_{\phi} f=P(\phi f)$, where $\phi$ is a bounded measurable function on the unit circle $\mathbb{T}=\partial \mathbb{D}$ and $P$ is the Szegö projection from $L^{2}$ (of the unit circle) to $H^{2}$. McDonald and Sundberg [4] defined Toeplitz operators on the Bergman space $A^{2}$ analogously: $\phi$ is a function on the interior of the disk and $P$ is the Bergman projection from $L^{2}(d A)$ ( $d A$ being area measure) to $A^{2}$.

In the Bergman space one can have $\phi f \in L^{2}$ for all $f \in A^{2}$ even if $\phi$ is unbounded. Moreover, the formula for the Bergman projection as an integral can be applied even when the product $\phi f$ is only in $L^{1}$. Given that, one quickly realizes that the formula for the Toeplitz operator

$$
P(\phi f)(z)=\int \frac{\phi(w) f(w)}{(1-\bar{w} z)^{2}} d A(w)
$$

allows one to extend the notion of Toeplitz operators to symbols that are measures (or even compactly supported distributions): simply replace $\phi d A$ with $d \mu$ in the formula (or apply the distribution to the appropriate product). This author [3] determined necessary and sufficient conditions on a positive measure $\mu$ for $T_{\mu}$ to belong to the Schatten classes $\mathcal{S}_{p}$. For complex measures the conditions were only sufficient. The same is true for the characterization of finite rank operators $T_{\mu}$ : necessary and sufficient conditions for positive measures were obtained, only sufficient for complex measures. The current author rashly claimed a characterization of finite rank for nonpositive measures, but the alleged proof was seriously flawed.

Lately, several researchers have shown an interest in obtaining a correct proof. Here, finally, one can be presented.

Received by the editors January 4, 2007 and, in revised form, February 21, 2007.

2000 Mathematics Subject Classification. Primary 46E20.

Key words and phrases. Bergman space, Toeplitz operator.

(c) 2007 American Mathematical Society 1717 
The characterization obtained here (that $\mu$ must be a finite sum of point masses) proves the conjecture that for $\phi \in L^{\infty}, T_{\phi}$ has finite rank only if it is 0 . After this paper was submitted, I learned of partial results on this conjecture in a preprint by A. Pushnitski, G. Rozenblum and N. Shirokov. They imposed some extra conditions on $\phi$. In addition, Namita Das communicated some incomplete work on the same conjecture.

I would like to express my thanks to the referee for suggestions to improve the exposition of the paper.

\section{The SETTING}

Initially, let $\mu$ be any complex regular Borel measure on the unit disk $\mathbb{D}$ in the complex plane $\mathbb{C}$. Integration with respect to area measure is denoted with $d A$. The set of all analytic functions on $\mathbb{D}$ will be denoted $\mathcal{H}(\mathbb{D})$ or simply $\mathcal{H}$.

The Bergman space $A^{2}$ of the unit disk is the space of all functions analytic in $\mathbb{D}$ which belong to $L^{2}=L^{2}(d A)$, that is, $A^{2}=L^{2} \cap \mathcal{H}$. The inner product in $L^{2}$ is denoted $\langle f, g\rangle=\frac{1}{\pi} \int f(z) \overline{g(z)} d A(z)$ and the corresponding norm is denoted $\|f\|=\langle f, f\rangle^{1 / 2}$. The Bergman kernel is the function $K(z, w)=K_{w}(z)=(1-\bar{w} z)^{-2}$. It satisfies $P f(w)=\left\langle f, K_{w}\right\rangle$ for all $f \in L^{2}$ where $P$ is the orthogonal projection from $L^{2}$ to $A^{2}$. In particular, if $f \in A^{2}$, then $f(w)=\left\langle f, K_{w}\right\rangle$. by

The Toeplitz operator on $A^{2}$ with symbol $\mu$ is denoted $T_{\mu}$ and is formally defined

$$
T_{\mu}(f)(w)=\frac{1}{\pi} \int_{\mathbb{D}} \frac{f(z)}{(1-\bar{z} w)^{2}} d \mu(z) .
$$

If $\mu$ has the form $\phi d A$ for some bounded measurable function $\phi$, then $T_{\mu}$ is denoted $T_{\phi}$ and satisfies $T_{\phi} f=P(\phi f), f \in A^{2}$. For arbitrary measures on $\mathbb{D}, T_{\mu}$ may be only densely defined because the integral (2.1) can only be guaranteed to converge for bounded $f$. Even if it converges, the result need not be in $A^{2}$. We will view $T_{\mu}$ as an operator defined on the dense subset of polynomials with range in the set of all analytic functions on $\mathbb{D}$. The question of when $T_{\mu}$ extends to $A^{2}$ or has values in $A^{2}$ will not be considered here. However, we note that if $|\mu|$ is a Carleson measure for $A^{2}$, then it is always true that $T_{\mu}$ is bounded from $A^{2}$ to $A^{2}$. In particular this is true for measures $\mu$ whose support is a compact subset of $\mathbb{D}$ as well as for measures of the form $\phi d A$ with $\phi$ bounded.

The following is the main theorem, whose proof will occupy the majority of this paper.

Theorem A. The rank of $T_{\mu}$ is finite if and only if $\mu$ is a finite linear combination of point masses.

One direction of this is obvious: if $\mu$ is a finite sum of point masses at points $z_{n}$, then the range of $T_{\mu}$ is the span of the finite set of functions $K_{z_{n}}$.

\section{SOME BACKGROUND}

The Bergman space setting is completely unnecessary and we will actually prove a theorem about operators on the space of analytic polynomials. Moreover, a large part of our proof does not require $\mu$ to be a measure. We have, formally, $\left\langle T_{\mu} f, g\right\rangle=\int f \bar{g} \mu$. For this to be true in the strict sense of the definition of $\langle\cdot, \cdot\rangle$, we would need to justify the implied exchange of integrals. What is clear, however, is 
that if $\mu$ is a measure on $\mathbb{D}$, then $T_{\mu} f$ will always produce an analytic function in $\mathbb{D}$. If $\mu$ is a measure on any disk, then we can use the same formula for $T_{\mu} f$ and obtain a function analytic in some neighborhood of 0 . The coefficients of any formal power series determine a linear functional on the space of polynomials in $\bar{z}$ in a standard way. If we interpret $T_{\mu} f$ in this way it is easy to prove that $T_{\mu} f(\bar{g})=\int f \bar{g} d \mu$. Thus $T_{\mu}$ can always be seen as taking polynomials to linear functionals on the conjugate analytic polynomials and $\mu$ can be seen as a linear functional on the space of polynomials in $z$ and $\bar{z}$. Moreover, these two objects determine each other. We now generalize these observations.

Let $\mathcal{P}$ denote the algebra of complex polynomials over $\mathbb{C}$ in the variable $z$ and let $\overline{\mathcal{P}}$ denote the polynomials in $\bar{z}$. Both are subalgebras of $\mathbb{C}[z, \bar{z}]$, the polynomials in both variables. Let $\mu$ be a linear functional on $\mathbb{C}[z, \bar{z}]$ and let $B_{\mu}(f, g)=\mu(f \bar{g})$. Let $T_{\mu} f$ denote the linear functional on $\overline{\mathcal{P}}$ defined by $T_{\mu} f(\bar{g})=B_{\mu}(f, g)=\mu(f \bar{g})$. One can determine the nature of $\mu$ by defining a topology on $\mathbb{C}[z, \bar{z}]$ and requiring that $\mu$ be continuous in that topology. For example, if $\mathbb{C}[z, \bar{z}]$ is given the topology of uniform convergence on compact sets, then a continuous $\mu$ can be identified with a complex measure with compact support. Compactly supported distributions come from the topology of uniform convergence on compact sets of all derivatives. Continuity in the $L^{1}(\mathbb{D}, d A)$ norm implies a bounded measurable function. We will need the exact nature of $\mu$ only in the last stages of our proof.

If the operator $T_{\mu}$ has rank less than $N$, then if we select $N$ polynomials $f_{j}$, there will exist a nontrivial linear relation

$$
\sum_{j=1}^{N} c_{j} T_{\mu} f_{j}=0 .
$$

If we apply these functionals $T_{\mu} f_{j}$ to polynomials $\bar{g}_{i}, 1 \leq i \leq N$, we obtain a set of column vectors in $\mathbb{C}^{N}$ that satisfies a linear relation with the same constants as (3.1). Thus, the matrix whose $i, j$ entry is $\mu\left(f_{j} \bar{g}_{i}\right)$ has a determinant equal to 0 .

The determinant is linear in each column and $\mu$ is a linear functional, so we can write

$$
\mu\left(f_{1}(z) \cdot \mid \begin{array}{cccc}
\overline{g_{1}(z)} & \mu\left(f_{2} \bar{g}_{1}\right) & \ldots & \mu\left(f_{N} \bar{g}_{1}\right) \\
\overline{g_{2}(z)} & \mu\left(f_{2} \bar{g}_{2}\right) & \ldots & \mu\left(f_{N} \bar{g}_{2}\right) \\
\vdots & \vdots & \ddots & \vdots \\
\overline{g_{N}(z)} & \mu\left(f_{2} \bar{g}_{N}\right) & \ldots & \mu\left(f_{N} \bar{g}_{N}\right)
\end{array}\right)=0 .
$$

Let us introduce the variable $z_{1}$ in place of $z$ above and use $\mu_{1}$ for $\mu$ acting in the variable $z_{1}$. Now we repeat this process in each column (using the variable $z_{j}$ in column $j$ and the notation $\mu_{j}$ for $\mu$ acting in $z_{j}$ ) to obtain

$$
\mu_{1}\left(\mu_{2}\left(\ldots \mu_{N}\left(\prod_{k=1}^{N} f_{k}\left(z_{k}\right) \operatorname{det}\left(g_{i}\left(z_{j}\right)\right)\right) \ldots\right)\right)=0 .
$$

We now specialize to the case where each $g_{i}$ has the form $g_{i}(z)=z^{k_{i}}$ with $k_{1}<$ $k_{2}<\cdots<k_{N}$. Let $J=\left(k_{i}\right)$ denote any such increasing $N$-tuple of nonnegative integers. Write $Z$ for the $N$-tuple $\left(z_{1}, z_{2}, \ldots, z_{N}\right)$ and write $V_{J}(Z)$ for the determinant $\operatorname{det}\left(z_{j}^{k_{i}}\right)$. Taking finite sums of equations (3.2), we get for any polynomial $F(Z)$ in $N$ variables:

$$
\mu^{N}\left(F(Z) \overline{V_{J}(Z)}\right)=0
$$


where $\mu^{N}$ is our abbreviation for successive applications of $\mu$ in each variable.

We now determine what one gets when we take linear combinations of $V_{J}$ with varying $J$ in this equation. We claim one gets

$$
\mu^{N}(F(Z) \overline{G(Z)})=0
$$

for all polynomials $F$ and all antisymmetric polynomials $G$. We now digress for a short discussion of symmetric and antisymmetric polynomials.

\section{Symmetric And Antisymmetric POLYNOMials}

A polynomial $F(Z)$ is called symmetric if it is invariant under permutations of the variables $z_{j}$, that is, $F(\pi(Z))=F(Z)$, where $\pi(Z)$ is the $N$-tuple consisting of the permutation of the coordinates of $Z$. We call $G(Z)$ antisymmetric (or alternating) if it changes sign with each transposition of coordinates. That is, $G(\pi(Z))=\epsilon_{\pi} G(Z)$, where $\epsilon_{\pi}$ is +1 for an even permutation $\pi$ and -1 for an odd $\pi$.

Denote by $S F(Z)$ and $A F(Z)$ the symmetric and antisymmetric projections of a function $F$. That is,

$$
S F(Z)=\frac{1}{N !} \sum_{\pi} F(\pi(Z)) \text { and } A F(Z)=\frac{1}{N !} \sum_{\pi} \epsilon_{\pi} F(\pi(Z)),
$$

where each sum is over all permutations. For any polynomial $F, S F$ is symmetric and $A F$ is antisymmetric. If $F$ is symmetric and $G$ is antisymmetric, then $S F=F$, $A F=0, S G=0$ and $A G=G$.

We observe that the vector space of all antisymmetric polynomials is the range of $A$ and is therefore the span of the images of all monomials. If $G(Z)=Z^{J}=$ $z_{1}^{k_{1}} z_{2}^{k_{2}} \ldots z_{N}^{k_{N}}$ is a monomial, then $A G(Z)$ is easily seen to be 0 if any of the exponents are equal. Moreover, if the monomial $G^{\prime}$ is obtained from the monomial $G$ by a permutation of the exponents, then $A G^{\prime}(Z)= \pm A G(Z)$. Thus, the set of antisymmetric polynomials is spanned by $A\left(Z^{J}\right)$ as $J$ varies over increasing $N$-tuples of nonnegative integers. It follows easily from the formula for the determinant as a signed sum of products that $A\left(Z^{J}\right)=V_{J}(Z) / N$ !. Thus, summing equations (3.3) produces equation (3.4), as claimed.

If $J=(0,1,2, \ldots, N-1)$, then $V_{J}(Z)=V(Z)$ is the Vandermonde determinant. Clearly the product of a symmetric polynomial and $V(Z)$ is antisymmetric. Ultimately, we will only need the fact that these products are in the range of $A$. However, the argument of the following paragraph shows that every antisymmetric polynomial is in fact the product of a symmetric polynomial and $V(Z)$.

The Vandermonde determinant $V(Z)$ is the minimal-degree polynomial $G(Z)$ vanishing on all the varieties $\mathcal{V}_{i, j}=\left\{Z: z_{i}=z_{j}\right\}$ for all pairs of indices $(i, j)$ with $i \neq j$. Therefore the ideal generated by $V(Z)$ is a radical ideal, and, by the Hilbert Nullstellensatz (see for example [2, Chapter 10, Theorem 2. 5]), any other polynomial vanishing on $\bigcup_{(i, j)} \mathcal{V}_{i, j}$ is divisible by $V(Z)$. It is clear that any antisymmetric polynomial $G(Z)$ vanishes on $\bigcup_{(i, j)} \mathcal{V}_{i, j}$ and hence $G(Z)$ is divisible by $V(Z)$.

This fact that every antisymmetric polynomial is divisible by $V(Z)$ is known. It is stated in the Encyclopedic Dictionary of Mathematics [1, Section 334. I], but it has been hard to find a published proof. 


\section{Proof of the Main theorem}

Let us recall that the following equation from section 3 :

$$
\mu^{N}\left(F(Z) \overline{V_{J}(Z)}\right)=0
$$

implies that for all polynomials $F$,

$$
\begin{gathered}
\mu^{N}(F(Z) \overline{G(Z)})=0 \quad \text { for all antisymmetric } G, \text { and so } \\
\mu^{N}(F(Z) \overline{H(Z) V(Z)})=0 \text { for all symmetric } H .
\end{gathered}
$$

Specializing to $F$ of the form $F_{1} V$ with $F_{1}$ symmetric gives us

(5.1) $\mu^{N}\left(F_{1}(Z) \overline{F_{2}(Z)}|V(Z)|^{2}\right)=0 \quad$ for all symmetric polynomials $F_{1}$ and $F_{2}$.

Now is the time to use the fact that $\mu$ is a measure, and to require that it have compact support. Let us restate our main theorem in the form of the ideas we have been using. In this form it is actually more general.

Theorem A (Restated). Let $\mu$ be a measure on $\mathbb{C}$ with compact support. Let $T_{\mu}$ be the operator from $\mathcal{P}$ to linear functionals on $\overline{\mathcal{P}}$ by $T_{\mu} f(\bar{g})=\int f \bar{g} d \mu$. Then $T_{\mu}$ has finite rank if and only if the support of $\mu$ is finite.

In the present case, $\mu^{N}$ is just a product measure on $\mathbb{C}^{N}$. We formally state our conclusions thus far in the language of measures and integration:

Proposition 5.1. If $T_{\mu}$ has rank less than $N$, then for all symmetric polynomials $F_{1}$ and $F_{2}$

$$
\int_{\mathbb{C}^{N}} F_{1}(Z) \overline{F_{2}(Z)}|V(Z)|^{2} d \mu^{N}(Z)=0 .
$$

It is clear that finite sums of products of the form $F_{1}(Z) \overline{F_{2}(Z)}$ (with $F_{1}$ and $F_{2}$ symmetric) form an algebra $\mathcal{A}$ of functions on $\mathbb{C}$ which contains the constants and is closed under conjugation. It doesn't separate points because each element is constant on sets of points that are permutations of one another. Define an equivalence relation $\sim$ on $\mathbb{C}^{N}$ by the fact that $Z_{1} \sim Z_{2}$ if and only if $Z_{2}=\pi\left(Z_{1}\right)$ for some permutation $\pi$. Let $Z=\left(z_{1}, \ldots, z_{N}\right)$ and $W=\left(w_{1}, \ldots, w_{N}\right)$. If $Z \not W$, then the polynomials $p(t)=\prod\left(t-z_{j}\right)$ and $q(t)=\prod\left(t-w_{j}\right)$ have different zeros (or the same zeros with different multiplicities). This implies that the coefficient of some power of $t$ in $p(t)$ differs from the corresponding coefficient in $q(t)$. Thus there is an elementary symmetric function that differs at $Z$ and $W$. Consequently, $\mathcal{A}$ separates equivalence classes.

Let us give the quotient space $\mathbb{C}^{N} / \sim$ the standard quotient space topology. If $K$ is any compact set in $\mathbb{C}^{N}$ that is invariant with respect to $\sim$, then $K / \sim$ is compact and Hausdorff. Also, any symmetric continuous function on $\mathbb{C}^{N}$ induces a continuous function on $\mathbb{C} / \sim$ (and conversely). Thus we can apply the Stone-Weierstrass theorem (on $K / \sim$ ) to conclude that $\mathcal{A}$ is dense in the space of continuous symmetric functions, in the topology of uniform convergence on any compact set. Therefore, for any continuous symmetric function $f(Z)$

$$
\int_{\mathbb{C}^{N}} f(Z)|V(Z)|^{2} d \mu^{N}(Z)=0
$$


If $f$ is an arbitrary continuous funtion, the above integral will be the same as the corresponding integral with $S f$ replacing $f$. This is because the function $|V(Z)|^{2}$ and the product measure $\mu^{N}$ are both invariant under permutations of the coordinates. We conclude that this integral vanishes for any continuous $f$ and so the measure $|V(Z)|^{2} d \mu^{N}(Z)$ must be zero. Thus, $\mu^{N}$ is supported on the set where $V$ vanishes.

This means $\mu$ must have fewer than $N$ points in its support: for if $z_{j}$ are $N$ distinct points in the support of $\mu$, then the point $Z=\left(z_{1}, \ldots, z_{N}\right)$ is in the support of $\mu^{N}$ but $V(Z) \neq 0$, a contradiction.

In fact, when the number of points in the support is finite, it is precisely the rank of $T_{\mu}$ : if the support of $\mu$ is $\left\{z_{1}, z_{2}, \ldots, z_{M}\right\}$, then the range of $T_{\mu}$ contains the $M$ independent evaluation functionals.

Note that the rank zero case has been known for at least a century: $\int f \bar{g} d \mu=0$ for all polynomials $f$ and $g$ clearly implies $\mu=0$ by the Stone-Weierstrass theorem.

\section{Some CONSEQUENCES}

Let $X$ be any subspace of $A^{2}$ with finite codimension. Let $S$ be the closure of $\left\{\sum f_{j} \bar{g}_{j}: f_{j} \in X, g_{j} \in A^{2}\right\}$ in the topology of uniform convergence on compact sets. Suppose $S$ is not all of $C(\mathbb{D})$; then there exists a measure $\mu$ with compact support in $\mathbb{D}$ such that $\int f \bar{g} d \mu=0$ for all $f \in S$ and all $g \in A^{2}$. That is, the range $Y_{\mu}$ of $T_{\mu}$ is contained in $X^{\perp}$, a finite dimensional set. This implies that $\mu$ is a finite sum of point masses and so $Y_{\mu}$ is spanned by the set of $K_{a}$ for $a$ in the support of $\mu$. If we repeat this for all possible measures that annihilate $S$ we get a set $E$ of all such points $a$. Then $E$ is finite because the corresponding $K_{a}$ are all independent and in $X^{\perp}$. Therefore, $S$ has finite codimension and contains all functions that vanish on $E$.

Also, $f \in X$ implies $\left\langle f, K_{a}\right\rangle=0$ for all $a \in E$ so all the functions in $S$ vanish on $E$. Thus $S$ is the ideal of all functions vanishing on $E$. This gives us the following corollary.

Corollary 6.1. If $X$ is a subspace of $A^{2}$ with finite codimension, then the closure of the span of $X \overline{A^{2}}$ in the topology of uniform convergence on compact sets is an ideal in $C(\mathbb{D})$ with a finite zero set. If $X$ has no common zeros, it is all of $C(\mathbb{D})$.

Note that it is not clear a priori that the closed span of $X \overline{A^{2}}$ is even closed under multiplication.

One can define operators to which our results apply that seem to have little to do with Toeplitz operators and nothing to do with Bergman spaces. For example, let $\mu$ be a measure on $\overline{\mathbb{D}}$ and define an operator from (say) the disk algebra to entire functions by $S_{\mu}(f)(w)=\int \exp (\bar{z} w) f(z) d \mu(z)$. Then, since $\exp (\bar{z} w)$ is a reproducing kernel for some appropriate normalization of the Fock space, one obtains $\left\langle S_{\mu}(f), g\right\rangle=\int f \bar{g} d \mu$ for all polynomials $f$ and $g$. If $S_{\mu}$ has finite rank, then $\mu$ must have finite support.

\section{REFERENCES}

1. Shôkichi Iyanaga and Yukiyosi Kawada (eds.), Encyclopedic Dictionary of Mathematics, Mathematical Society of Japan, 1977, english edition: MIT Press, Cambridge, MA, 1980. MR591028 (84a:00015)

2. Serge Lang, Algebra, second ed., Springer-Verlag, New York, 1984. MR1878556 (2003e:00003) 
3. Daniel H. Luecking, Trace ideal criteria for Toeplitz operators, J. Funct. Anal. 73 (1987), 345-368. MR899655 (88m:47046)

4. Gerard McDonald and Carl Sundberg, Toeplitz operators on the disc, Indiana Univ. Math. J. 28 (1979), no. 4, 595-611. MR542947 (80h:47034)

Department of Mathematical Sciences, University of Arkansas, Fayetteville, ArKANSAS 72701

E-mail address: luecking@uark.edu 